\title{
RESEARCH ON CONSTRUCTION OF SPATIO-TEMPORAL DATA VISUALIZATION PLATFORM FOR GIS AND BIM FUSION
}

\author{
DOU Shiqing, ZHANG Honghua,ZHAO Yanqin, WANG Aimin, XIONG Youtao, ZUO Jingmeng \\ College of Mining Engineering, Heilongjiang University of Science and Technology, \\ Harbin, 150022, China
}

KEY WORDS: spatio-temporal Data; multi-dimensional spatial visualization; smart city; GIS; BIM

\begin{abstract}
:
The visualization model of GIS and BIM fusion can provide data bearing platform and main technical support for future urban operation centers, digital twin cities, and smart cities. Based on the analysis of the features and advantages of GIS and BIM Fusion, this paper proposes a construction method of the spatio-temporal data visualization platform for GIS and BIM Fusion. It expounds and analyzes the overall architecture design of platform, multi-dimensional and multi-spatial scales visualization, space analysis for GIS and BIM fusion, and platform applications and so on. The urban virtual simulation spatio-temporal data platform project of Teda New District in Tianjin has verified and demonstrated that the effect of application is good. This provides a feasible solution for the construction of spatio-temporal Data Visualization Platform.
\end{abstract}

\section{INTRODUCTION}

The GIS industry has gradually developed from traditional electronic map display, query analysis, and data processing application to big data analysis, mining, and spatio-temporal data visualization, which is developing towards big data, cloud platform, distributed computing, multi-source data integration, etc., and presenting a trend of large scale, micro field and full content. In the era of big data, the GIS industry cannot ignore the temporal and spatial elements of data, so the spatio-temporal big data and management platform come into existence as the situation requires ${ }^{[1-3]}$.

BIM+GIS allows the exchange and interoperability between BIM information in the micro field and GIS information in the macro field, which has become a research hotspot in both academic and industrial circles ${ }^{[4-6]}$. The visualization mode based on GIS+BIM can support multi-level visualization of spatiotemporal big data platform, but the platform needs to support BIM and GIS to store and mine their large data sets ${ }^{[3]}$.

The GIS platform can be built based on the visualization requirements of spatiotemporal big data, and the urban scene can be built with technologies such as computer graphics, image processing, and human-computer interaction as well as $2 \mathrm{D}$ and $3 \mathrm{D}$ spatial geographic environments. Combined with business needs, various statistical graphs can be used to display spatial and temporal elements and analysis results. In addition, spatial analysis capability can be used to restore and even augment the global structure and specific details of spatiotemporal data so that the intrinsic value of spatiotemporal big data can be further mined [7-9]. Based on TEDA urban spatiotemporal big data platform, this research explored GIS+BIM for the building of spatiotemporal big data visualization platform.

\section{OVERALL ARCHITECTURE OF SPATIO-TEMPORAL BIG DATA VISUALIZATION PLATFORM}

The spatiotemporal big data platform is essentially GIS platform undertaking visual interaction, storing data, and executing other auxiliary functions in the project. Based on GIS+BIM technology, the platform displays visual information of TEDA, such as administrative boundaries of territory and BIM models of major buildings. As the carrier of city operation center and "digital twin city", the platform also provides an approach for urban 
space planning, emergency response, real-time [10-11].

information transmission, and urban problem solving

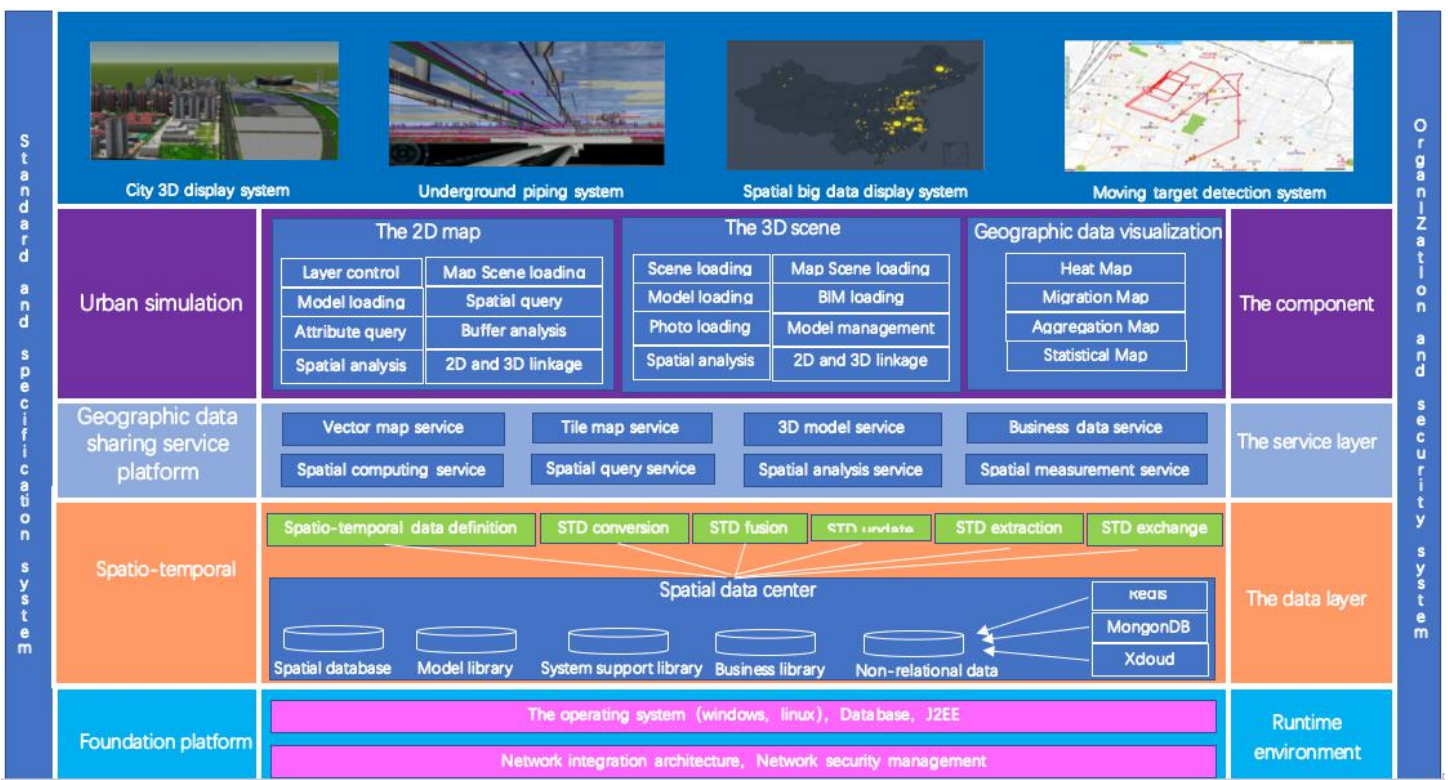

Fig.1 Overall architecture diagram of GIS development framework

The GIS development framework consists of urban simulation platform, spatiotemporal integration platform, geographic data sharing service platform, and geographic data visualization platform, and each of the four parts bears some functions of the overall GIS framework. Fig. 1 shows the overall architecture of the GIS development framework.

\subsection{Data layer based on spatiotemporal integration platform}

Spatiotemporal data is geospatial data with time tags, including both traditional geospatial data and unstructured data such as text with spatiotemporal tags. Considering diverse sources and formats of spatiotemporal big data, their mining and processing are important tasks for visual representation, because they define unified data management portal, establish spatiotemporal data storage standards, provide automated processing tools, and help complete data handling.

Based on the spatiotemporal big data platform, the exploratory storage is tested according to TEDA data, including BIM model, vector map, grid map, humanities, POI, urban real-time traffic, and weather, and other data that can be visualized and have certain value(seen Fig. 2). It is one of the capabilities of the platform to visualize and store the data based on
NoSQL database to meet the multi-model, multi-scale, and multi-dimensional structure of spatiotemporal big data.

The spatiotemporal integration platform achieves integrated management of spatiotemporal data, such as definition, extraction, storage, exchange, query, and analysis. It provides data spatiotemporal deduction, supports customized spatiotemporal data model, and realizes the basic operation of spatiotemporal data (add, edit, delete, etc.). It supports data exchange between traditional database and spatial database, query functions such as attribute query, time query, spatial query, fusion query, time measurement and spatiotemporal data integrated query, and spatiotemporal analysis functions such as smooth calculation, overlay analysis, spatiotemporal sequence analysis and predictive analysis. In addition, the platform provides spatiotemporal deduction of data in combination with its spatiotemporal characteristics.

\subsection{Service layer based on geographic data sharing platform}

With the geographic data sharing platform as the core, the spatial data service center is built to provide vector map service, tile map service, 3D model service, and business data service; combined with the characteristics of spatial data, it provides spatial 
computing services, such as projection transformation, spatial logic judgment, spatial query, buffer, and spatial measurement; combined with the characteristics of spatiotemporal data, it provides functions, such as spatiotemporal measurement, spatiotemporal prediction, spatiotemporal query, and spatiotemporal analysis; it authenticates services to ensure data security.

The geographic data sharing platform provides spatial location big data interface services, monitors mobile targets, replays historical running data, and utilizes geographic location big data for mining analysis; it provides tools related to spatial data operation, such as spatial logic operation, network analysis, and coordinate transformation; it supports management of geographic big data services, including map service, spatial element service, 3D model service, and spatial data query service; it supports access management of third-party data or map service as well as data extraction, cleaning, analysis, combination and handling to achieve data exchange between different departments.

2.3 Component layer based on urban simulation platform

The urban simulation platform provides spatial data display components, such as browser-based 2D map and 3D scene. These components developed by JavaScript can be used without plugins and seamlessly integrated with the browser. The core capabilities of these componets are as following: they can provide complete 2D map display framework, satisfy loading and display of multi-source map services, support basic operation of map, and achieve query and basic analysis of spatial data; they can provide urban 3D scene display interface, satisfy loading and display of 3D model and map services, and support data query and positioning, as well as $3 \mathrm{D}$ measurement and analysis based on 3D scenes, 2D and 3D linkage; they can display underground 3D pipeline, support management of 3D pipeline model, pipeline data query, and pipeline analysis, and support linkage of 2D map and $3 \mathrm{D}$ scene. Based on the $2 \mathrm{D}$ and $3 \mathrm{D}$ spatial data display components provided by the urban simulation platform, the application system is built to meet the needs of different industries.

\subsection{Application layer based on geographic data visualization platform}

As an important direction of big data research, visual expression of multidimensional spatiotemporal data is supported by rich product components that can reduce the threshold of application of spatiotemporal data and the learning cost of visual expression.

Combined with the business needs of different urban departments, the GIS application system developed on this platform provides technical support for the development of smart city application system; GIS application standardized products and application systems are built, such as moving target detection system, urban 3D scene display system, and urban underground pipeline management system.

\section{GIS+BIM VISUALIZATION UNDER SPATIOTEMPORAL BIG DATA PLATFORM}

\subsection{Visualization of multidimensional and multi-spatial scales}

The simple visualization mode is difficult to meet city-class visualization needs, because the rapidly developing urban space and environment are increasingly complicated and diverse from the former simple and clear hierarchy, which raises new requirements on the development of urban integration. In the case of emergencies, macroscopic, mesoscopic and microscopic visualization modes can be used specific to the coordinates of a certain corner of the city to divide a rational and scientific buffer. Therefore, according to project requirements, GIS+BIM can be used for city-class indoor and outdoor, above-ground and underground integration to realize the integration of TEDA spatiotemporal big data platform.

TEDA spatiotemporal big data platform achieves urban integration and visualizes the major buildings in the area. As shown in Fig.2, in order to macroscopically visualize TEDA, the white model (rough model) of the visible area is loaded based on oblique photogrammetric data to macroscopically 
The International Archives of the Photogrammetry, Remote Sensing and Spatial Information Sciences, Volume XLII-3/W10, 2020 International Conference on Geomatics in the Big Data Era (ICGBD), 15-17 November 2019, Guilin, Guangxi, China

display urban spatial geographic information such as the main outline of the city and the location information of the building. The data information is loaded and displayed according to the default city theme on the platform to complete the macroscopic visualization of TEDA. The macroscopic visualization mode provides path query, emergency command and rapid response route planning, scientific solutions for urban traffic congestion as well as urban spatial analysis and planning. From the macro perspective, a series of platform-based applications can be displayed, such as moving target detection, spatiotemporal data query and analysis, and assistant decision-making.

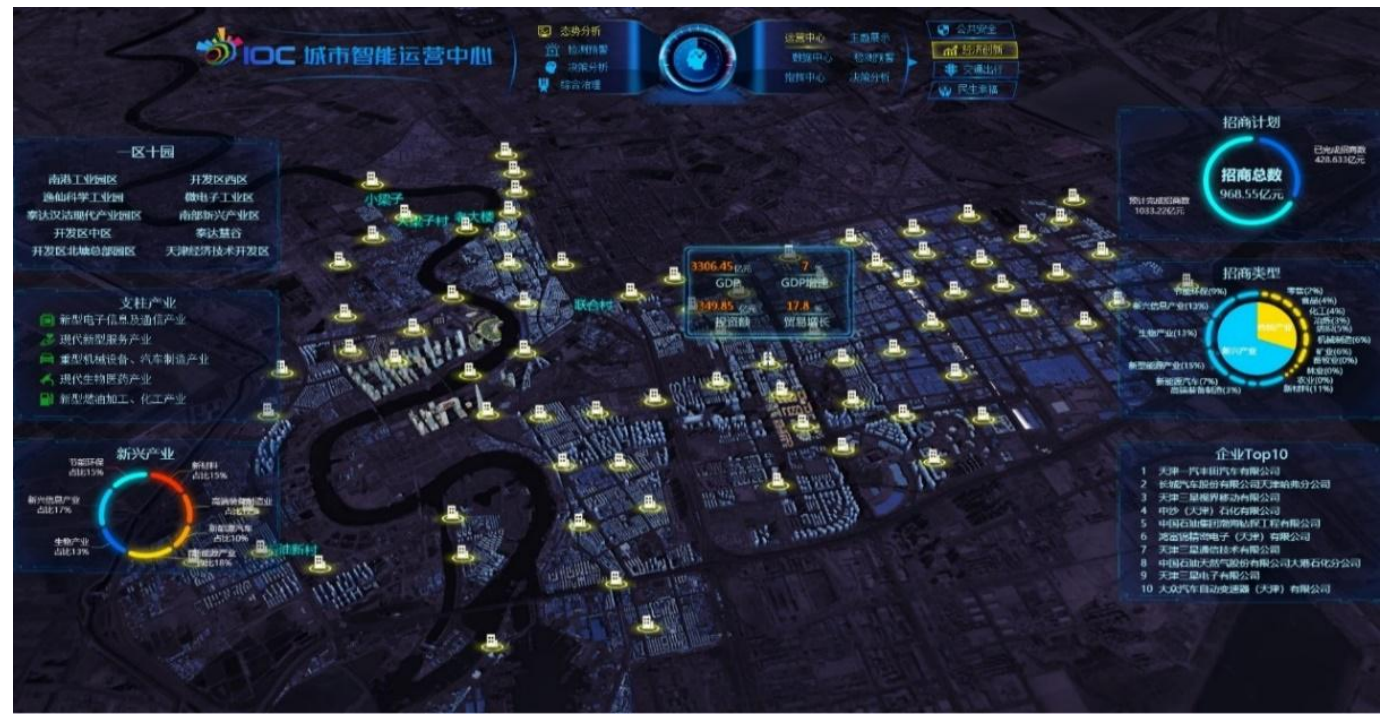

Fig.2 macro visualization

As a BIM model with shorter visual distance and finer load and display, the mesoscopic visualization mode can load the attribute data by clicking on the model data within the visible range, as shown in Fig.3. Medium visualization provides the view of major urban buildings, urban POI information based on the platform standard, customized route for urban roam, illumination, spatial topology and visibility. The multi-purpose BIM model of the platform can measure and display the target building from the mesoscopic perspective, and calculate the distance, area, latitude and longitude, so that natural effects and urban emergency effects can be achieved from the mesoscopic perspective.

and urban space analysis such as viewshed,

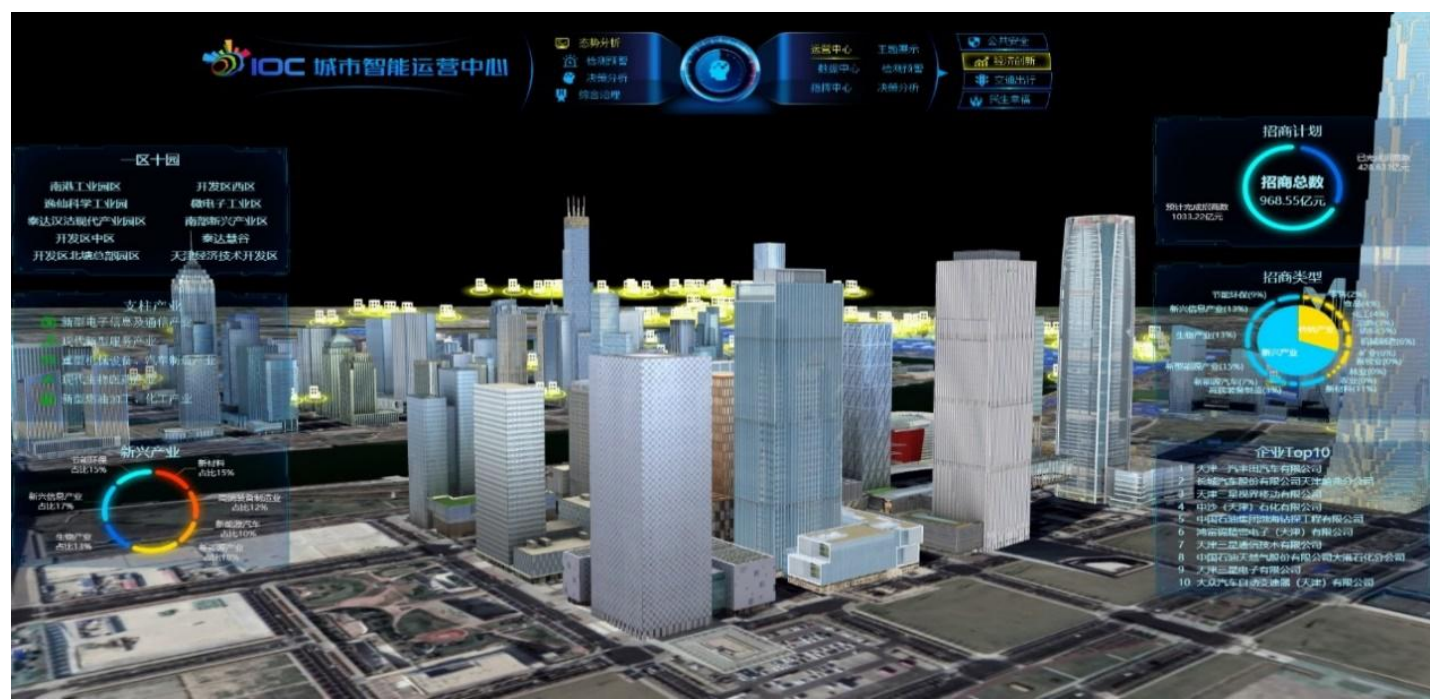

Fig.3 medium visualization 
Microscopic visualization based on the fine characteristics of the BIM model allows the browse of the inside of target buildings as required by the perspective. In this case, the surveillance camera can be placed and the camera at any floor can be selected during the browse so that the real-time surveillance picture can be displayed in the other two screens, As shown in Fig.4.

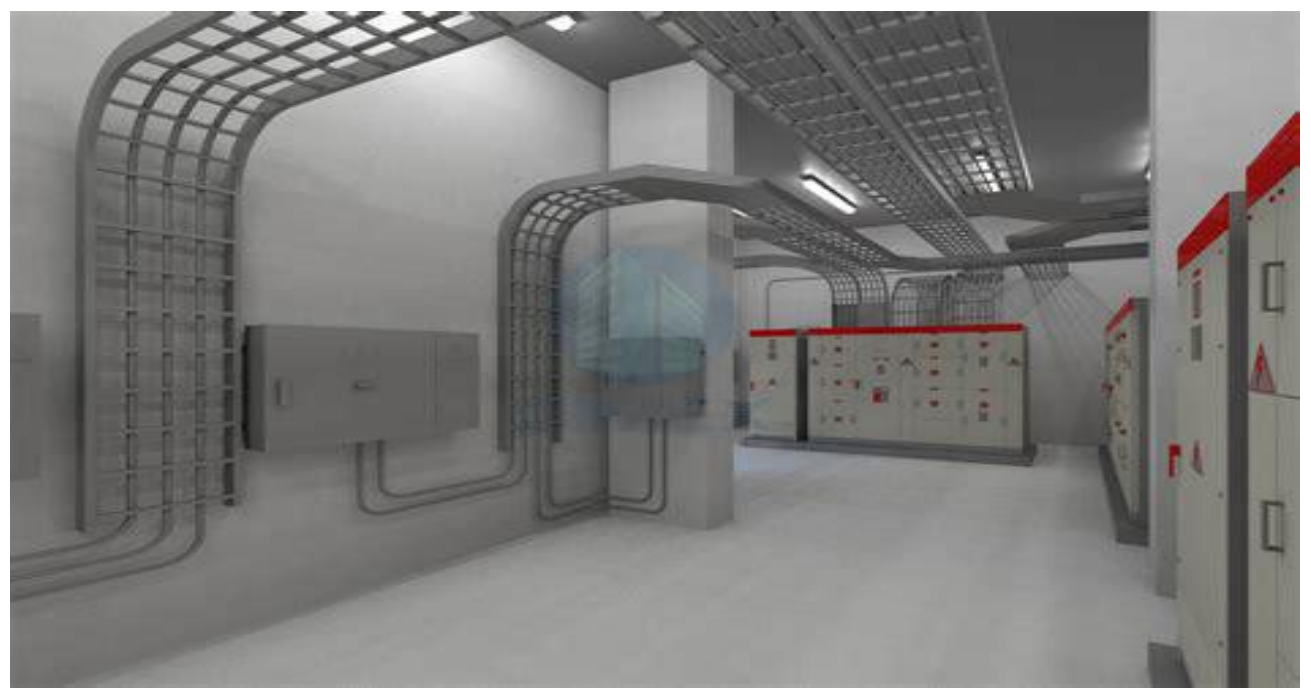

Fig.4 microscopic visualization

With the GIS+BIM visualization mode, TEDA spatiotemporal big data platform realizes multi-level and multi-dimensional urban visualization from macroscopic-mesoscopic-microscopic perspectives. Based on the platform, government agencies and relevant industries can control the city from the macroscopic, mesoscopic and microscopic perspectives and visualize the real-time data in urban space. With the improvement in platform construction, visualization analysis and decision-making based on spatiotemporal big data are likely to eventually become the basic bearing platform for other systems.

\subsection{Spatial analysis based on GIS+BIM}

The spatial analysis on the platform mainly relies on GIS means. Based on GIS+BIM urban building model data, mathematical algorithm and database technology are used for analytical calculation, data calculation and joint operation. With the spatial analysis function, the platform achieves capabilities such as urban spatial planning and analysis, urban emergency prediction, and visualization analysis. The functions that the platform has achieved are urban viewshed analysis, urban skyline drawing, building section analysis, conflict detection, flooding analysis, video placement, and spatial topology analysis (Fig.5 8).

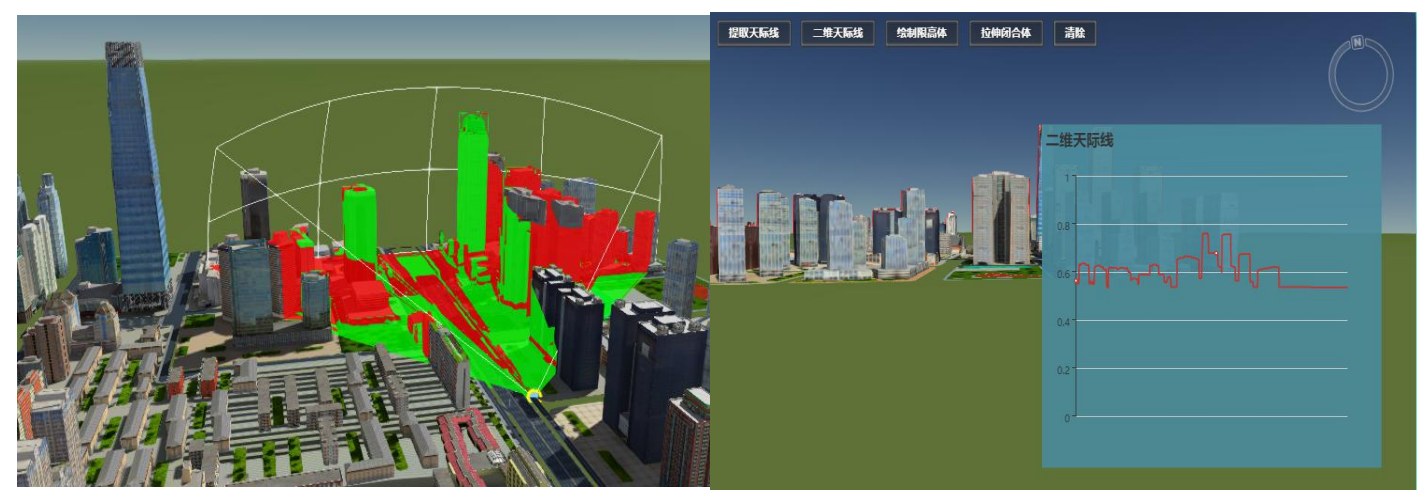

Fig.5 Visual analysis and mapping of the skyline 


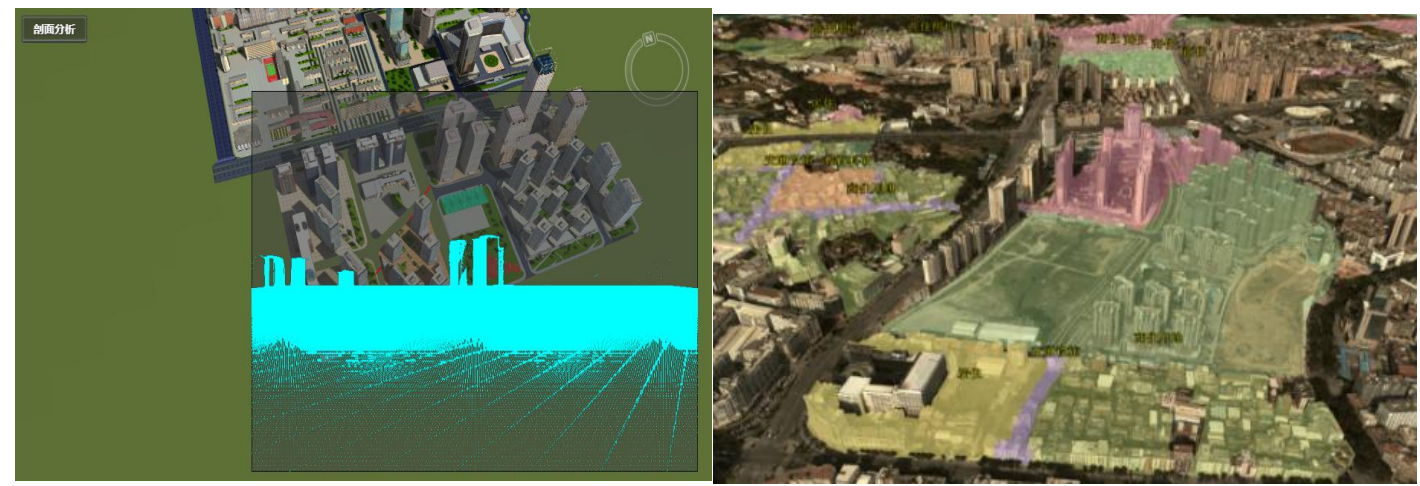

Fig.6 profile drawing and conflict detection

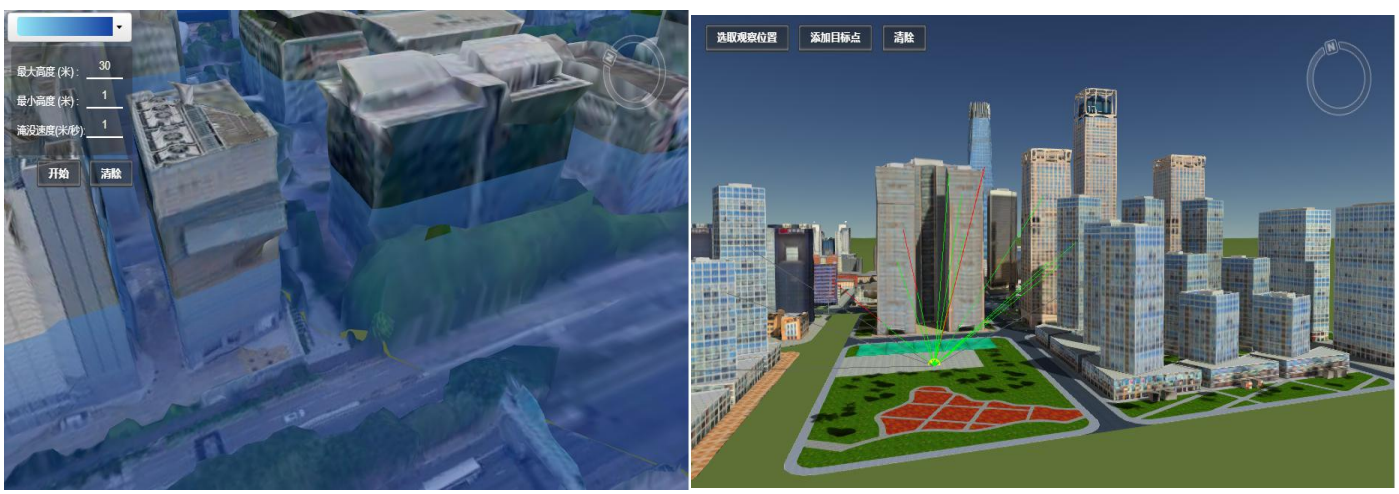

Fig.7. Inundation analysis and visibility analysis

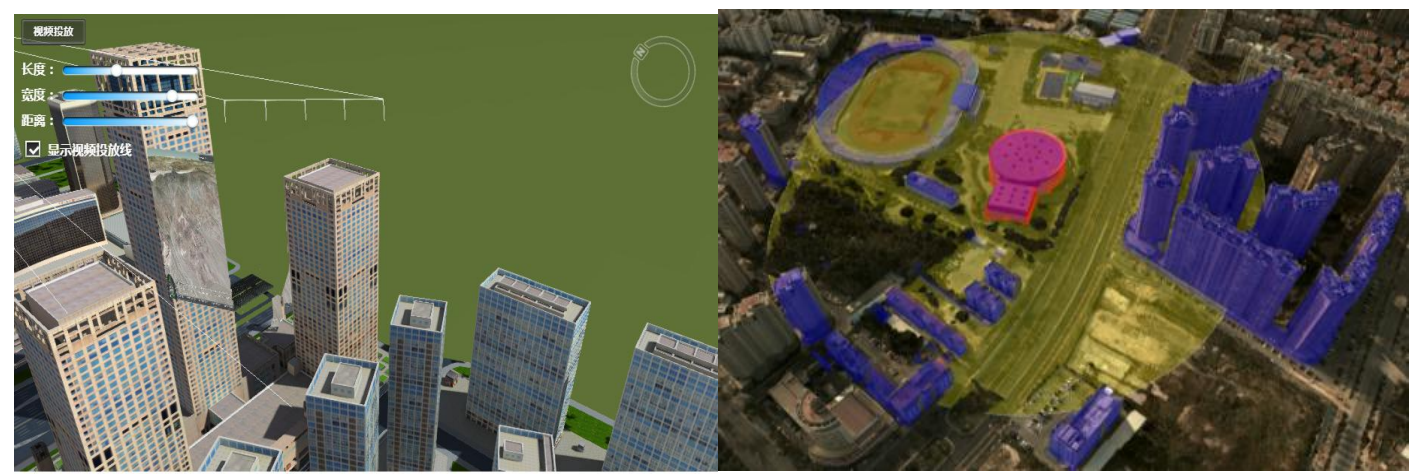

Fig.8 video placement and spatial topology analysis

\section{APPLICATION OF SPATIOTEMPORAL BIG} DATA PLATFORM

\subsection{Civil application}

The major task of TEDA spatiotemporal big data platform at this stage is urban integration facilitating the management and planning of governmental departments. The integration will rely on visualization technology to visualize buildings, urban geographic data, spatial attribute data, and city-related data
information(Fig.9). The major application at this stage is to partition the city based on GIS+BIM visualization mode to report events such as emergency, fire, and gas leakage. Based on its visualization capability, the platform can set floating labels for hospitals at all levels in the city, so that residents can view them based on the grids, including hospital environment, facilities, and physicians and select hospitals based on real-time traffic with a view to timely medical treatment. 


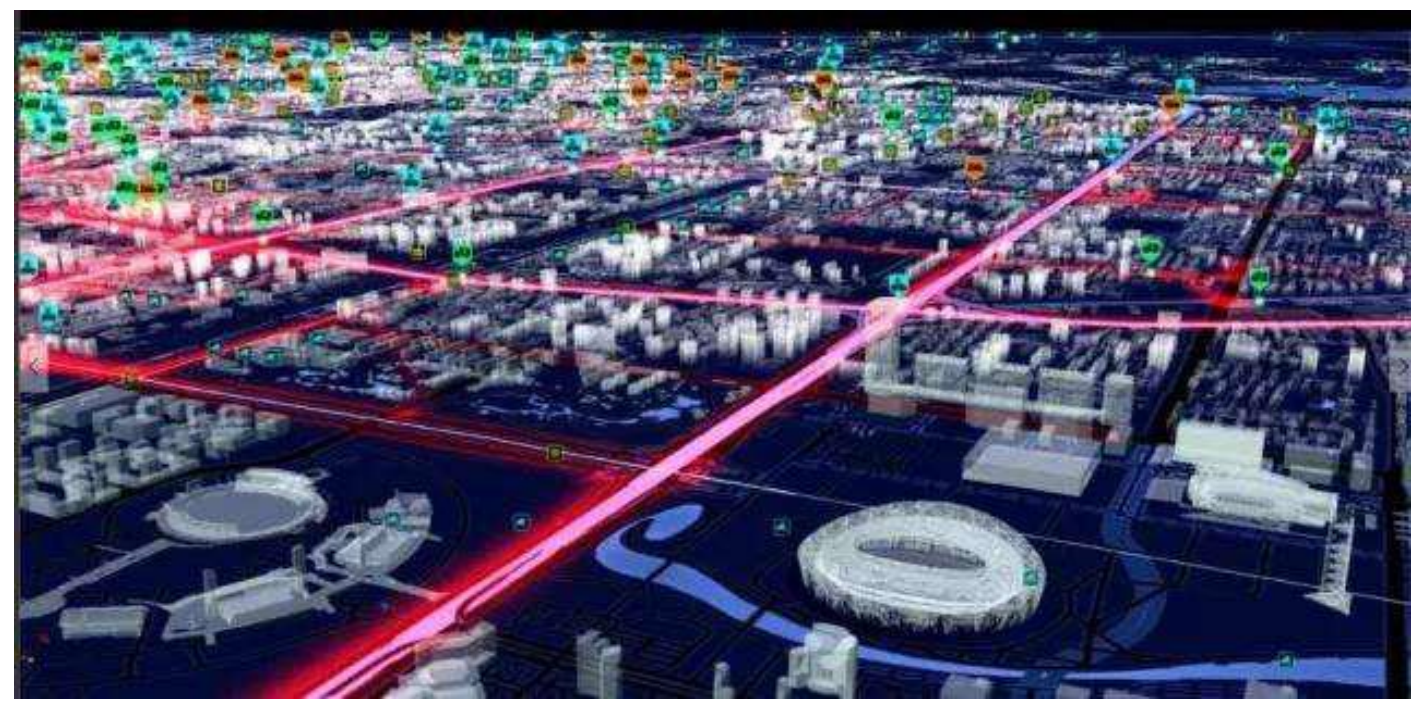

Fig.9 urban grid hospital visualization

\subsection{Social application}

The major social application that has been achieved by the platform is "the Internet of Things". The most prominent feature of the Internet of Things is the interconnection of things, which must be based on the GIS platform. The Internet of Things today is best reflected in the logistics information of express delivery, because the continuous technical progress makes it possible to update the logistics information in real time, which is the incomparable advantage and capability of the Internet of Things. However, these implementations are all based on the GIS platform.

\subsection{Governmental application}

As a new trend in recent years, the digital office saves many intermediate links and improves problem-solving efficiency of the government, especially for the departments of land, environment and agriculture, where the digital office is even more important, but this must be based on GIS technology (Fig.10) .

The departments of urban management and planning are facing difficulties on the increasingly complex environment and administrative areas, so unclear responsibilities will result in inefficient work and relevant departments cannot respond quickly to emergencies. The spatiotemporal big data platform can solve the above problems based on GIS+BIM grid management and emergency command. As shown in Fig.11.

Moving target detection technology has great potential of application in handling urban traffic congestion, which is a serious problem especially in some big cities, monitoring population, Supported by GIS+BIM visualization mode technology, real-time traffic conditions can be visualized with the traffic information uploaded by the sensor, which provides technical means for traffic scheduling or route planning for drivers according to the number of vehicles on each road. This technology can also assist public security organs to handle cases. After capturing the uploaded detailed information of fugitives, the sensor-based face recognition technology will use the multi-level visualization of the platform and urban sensors for motion detection and assist in arresting. It can also set an electronic fence for the range of activities based on electronic devices to protect children. 

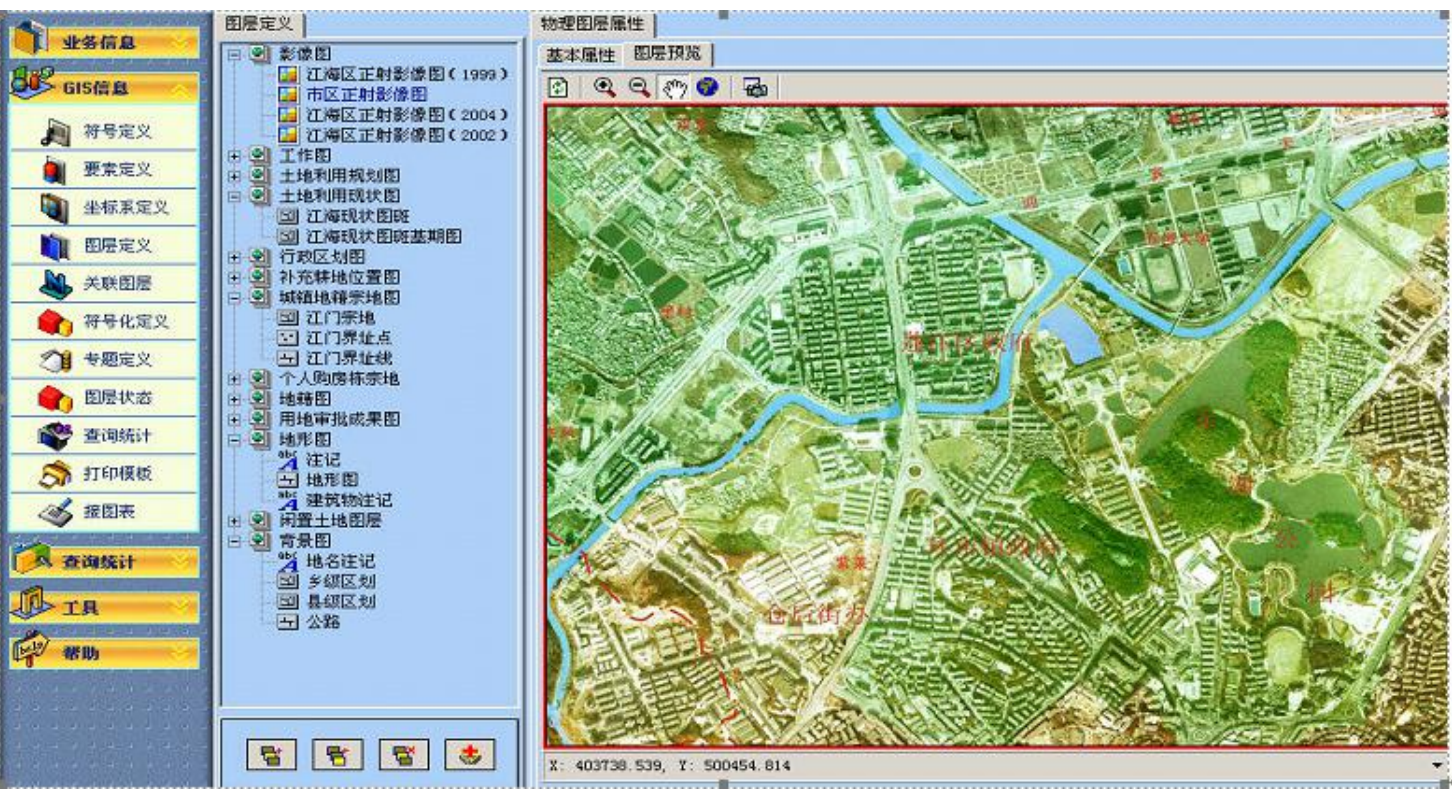

Fig.10 digital government

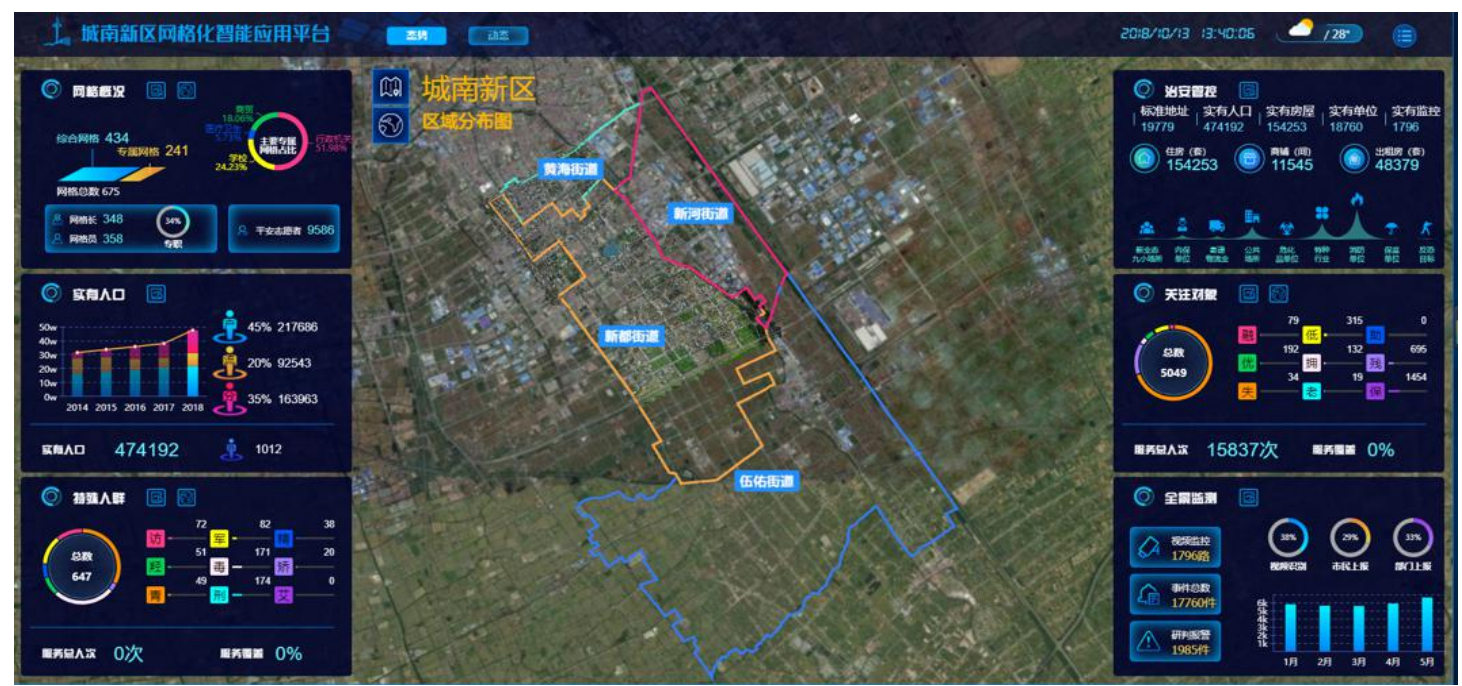

Fig.11 Grid and emergency command

\section{CONCLUSION}

Based on TEDA spatio-temporal big data visualization platform construction project, this research analyzed, introduced and explained the overall architecture of the spatiotemporal big data platform, showed the application of GIS+BIM, and achieved urban macroscopic, mesoscopic and microscopic visualization modes. In addition, it combined with urban application scenarios to explain GIS+BIM technology. The platform has achieved ideal application results, and the visualization analysis and control with multidimensional and multi-spatial scales realized by the spatiotemporal big data platform provide technical support for the development of urban intelligent operation center(IOC), "digital twin", and smart city. As a large-scale project with fundamentality and applicability, the spatiotemporal big data visualization platform follows the development trend of the Internet and big data, so its building is a necessary measure to improve the urban transformation and upgrading strategy and the national sustainable development policy, as well as promote urban quality and comprehensive competitiveness.

\section{REFERENCES}

[1]WANG Jiayao. Spatio-temporal big data and its 
application in smart cities[J]. Satellite Application,2017,03,10-17.

[2] LI Deren,YAO Yuan SHAO Zhenfeng. Big Data in Smart City[J].Geomatics and Information Science of Wuhan University, 2014,(39)06,631-640.

[3]. LI Deren,MAJun, SHAO Zhenfeng. Discussion on spatio-temporal big data and its application[J]. Satellite Application, 2015,09,7-11.

[4] Wang J , Hou L , Chong H Y, et al. A Cooperative System of GIS and BIM for Traffic Planning:A High-Rise Building Case Study[C]// International Conference on Cooperative Design, Visualization and Engineering. Springer, Cham, 2014.G

[5] Davidson K. Geodesign combines strengths of BIM and GIS in new convergence platfo-rms[J].ENR (Engineering News-Record), 2014, Vol.272(7)OCLC

[6] WU Pengfei, LIU Yuahen,TAN Yi, LI Jianfeng. Advances and Trends of Integration between GIS and BIM[J]. GEOMATICS \& SPATIAL INFORMATION TECHNOLOGY,2019,42(01):1-6.
[7] Ministry of Natural Resources of the People's Republic of China. Technical outline for the construction of smart city spatio-temporal big data platform (2019 edition) [K].

[8]. LIU Quanhai, RAN Huimin, LI Lou, et al. With the wisdom planning of space-time information intelligent collection platform construction $[\mathrm{J}]$. Technology Geographic Information, 2015(06):64-67. [9] XU Yizhi. Research on Technologies of spatiotemporal data pulsation analysis based on GIS platform[D].Hefei: The University of Chinese Academy of Sciences,2017.05.

[10] ZHOU Yu, LIU Chuncheng. The Logic and Innovation of Building Digital Twin City in Xiong'an New Area[J].URBAN DEVELOPMENT STUDIES, 2018,25(10):60-67.

[11] RAN Hui-min,PAN Boming,GUO Yunyan. Construction and Research of Time and Space Information Cloud Platorm[J]. Modern Surveying and Mapping, 2015(4):34-36. 